Diana Kapiszewski, Georgetown University

Sebastian Karcher, Syracuse University

Published as: Kapiszewski, Diana, and Sebastian Karcher. 2021. "Empowering Transparency: Annotation for Transparent Inquiry (ATI)." PS: Political Science \& Politics 54 (3): 473-78. https://doi.org/10.1017/S1049096521000287

\title{
Empowering Transparency: Annotation for Transparent Inquiry (ATI)
}

Political scientists generate, analyze, and deploy myriad types of qualitative data to support their claims and conclusions. To produce those data, they conduct interviews and focus groups, collect archival documents, download video clips, record music, take pictures of varied phenomena, and use many other techniques. Given the heterogeneity of the resulting data, and the different ways in which authors deploy them to support the arguments in their scholarship, authors who use such data face challenges in making their work more transparent.

Annotation for Transparent Inquiry (ATI) is a new approach to transparency in qualitative and multi-method research that addresses those challenges. ATI helps authors to demonstrate the rigor of their work, and to make their scholarship more comprehensible and evaluable, democratizing access to and fostering the accumulation of knowledge.

This symposium comprises brief pieces written by some of the scholars who have pioneered the use of ATI in political science. ${ }^{1}$ As the contributions demonstrate, ATI facilitates transparency in multiple types of qualitative inquiry, and in multi-method scholarship as well. Authors discuss how ATI strengthened their work, consider what difficulties and costs using ATI created, and propose best practices for using ATI; they also highlight new

1 A list of published articles that have been annotated using ATI can be found here: https://qdr.syr.edu/ati/ati-models. 
intellectual frontiers ATI can help scholars to reach with regard to both producing and consuming knowledge. In aggregate, the contributions suggest that ATI holds the potential to considerably increase the transparency, as well as the rigor and richness, of qualitative scholarship. While all contributors acknowledge that using ATI requires time and effort, they argue that - especially if scholars anticipate using ATI and plan accordingly - this effort is manageable and worthwhile.

\section{Research Transparency and ATI}

Research transparency - describing in detail how the data underlying a piece of scholarship were generated and analyzed, and sharing those data ethically and legally - is an important goal across scientific disciplines. Broad acceptance of the value of transparency springs from four key contributions it makes to the reliable production of new knowledge. First, open data and materials allow scholars to illustrate the power and rigor of their work. Second, transparency increases the clarity of scholarship, augmenting the comprehension of diverse readers, from scholars to policymakers to activists and other key political actors. Third and relatedly, transparency facilitates rigorous evaluation, allowing scholars to assess the limitations and recognize the strengths of others' research, potentially enhancing their confidence in its claims and conclusions. Fourth and most generally, transparency democratizes access to and promotes the accumulation of knowledge, encouraging and empowering scholars to build on each other's findings and re-use shared data in their research and teaching.

Recent investments in socio-technical infrastructure, i.e., capabilities, arrangements, and institutions arising from the interdependent relationship between social and technological systems (see Sawyer and Jarrahi 2014), have empowered unprecedented levels of transparency and addressed challenges in achieving it (Miguel et al. 2014; Elman, Kapiszewski, and Lupia 2018). In the social sciences, most of this new capacity has been directed to, and mainly facilitates transparency in, quantitative inquiry. Due to the varied forms that qualitative data take - i.e., text, images, audio, and video - and the way they are deployed in published work, scholars whose work entails generating and analyzing such data require different mechanisms and strategies to achieve transparency (Karcher et al. 2016; 
Mannheimer et al. 2019). ${ }^{2}$

Qualitative data are typically analyzed, and used to support claims in scholarship, individually or in small groups: an archival document, interview recording, newspaper article, video clip, or a small groups of such sources, serve as distinct inputs to an analysis. Yet increasingly stringent journal article word-count limits and publisher concerns about the length of book manuscripts make it difficult for authors to use large amounts of text as supporting material, thereby complicating the deployment of the very forms of evidence that are the lifeblood of much qualitative work. Further, rather than presenting results in a single matrix as authors of quantitative work do, authors who deploy qualitative data often interweave data, analysis, and conclusions across the span of their written work. Figure 1 compares how data are analyzed and deployed in quantitative vs. qualitative research.

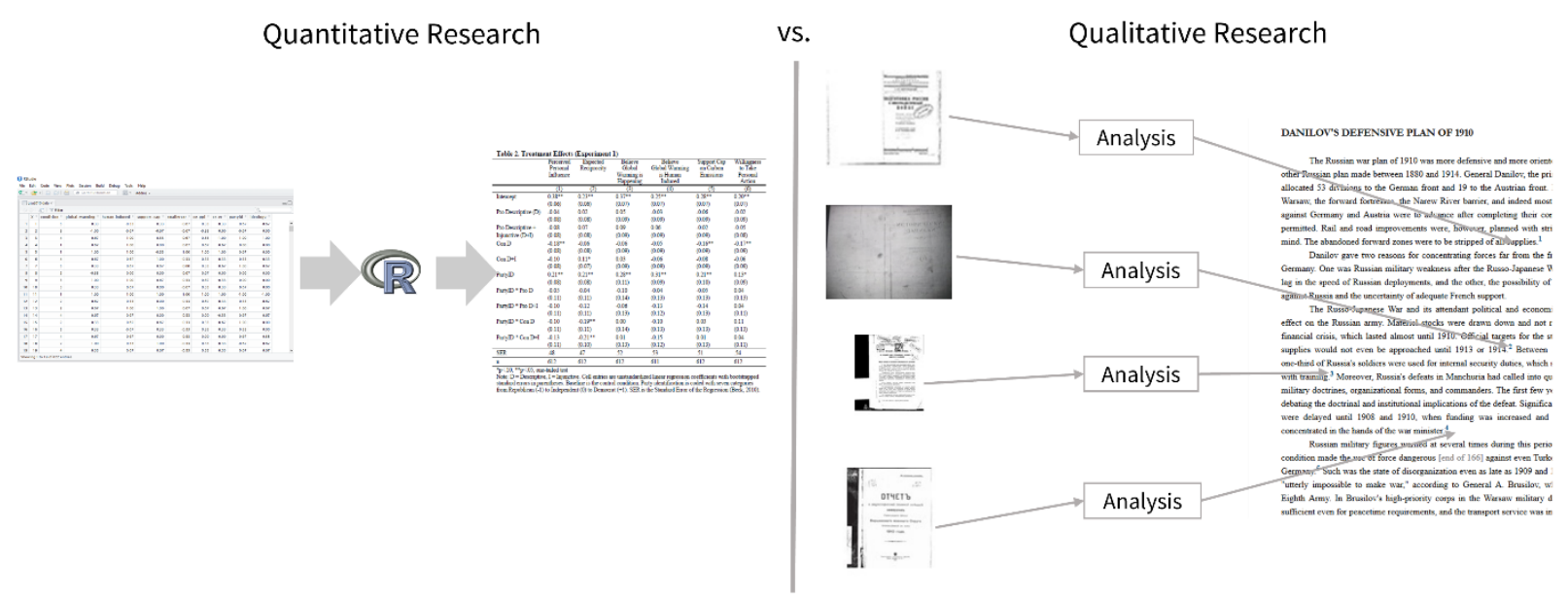

Figure 1: Data and analysis in quantitative vs. qualitative research. Quantitative analysis from Bolsen, Leeper, and Shapiro 2014; Qualitative analysis from Snyder 2015

Annotation for Transparent Inquiry (ATI) accommodates these features of qualitative inquiry. Developed by the Qualitative Data Repository (QDR) and the software non-profit Hypothesis, ATI is a flexible, versatile approach to achieving transparency in qualitative and

\footnotetext{
${ }^{2}$ The Qualitative Transparency Deliberations (QTD, https://www.qualtd.net/) productively considered these and other questions about how to make qualitative research more transparent.
} 
multi-method research.3 ATI employs open web annotation, which allows for the generation, sharing, and discovery of digital annotations across the web. Authors who use ATI digitally annotate particular claims or conclusions in their manuscript. Annotations may include full citations to the data sources underlying empirical assertions; "analytic notes" that clarify how the author generated or analyzed their data and/or how they support the author's inferences or interpretations; excerpts from data sources; and potentially links to the data sources themselves (see Figure 2). For scholars who use lengthy footnotes to clarify and illustrate points, ATI annotations are a natural extension of their writing practices (see also Gerring in the conclusion of this symposium).
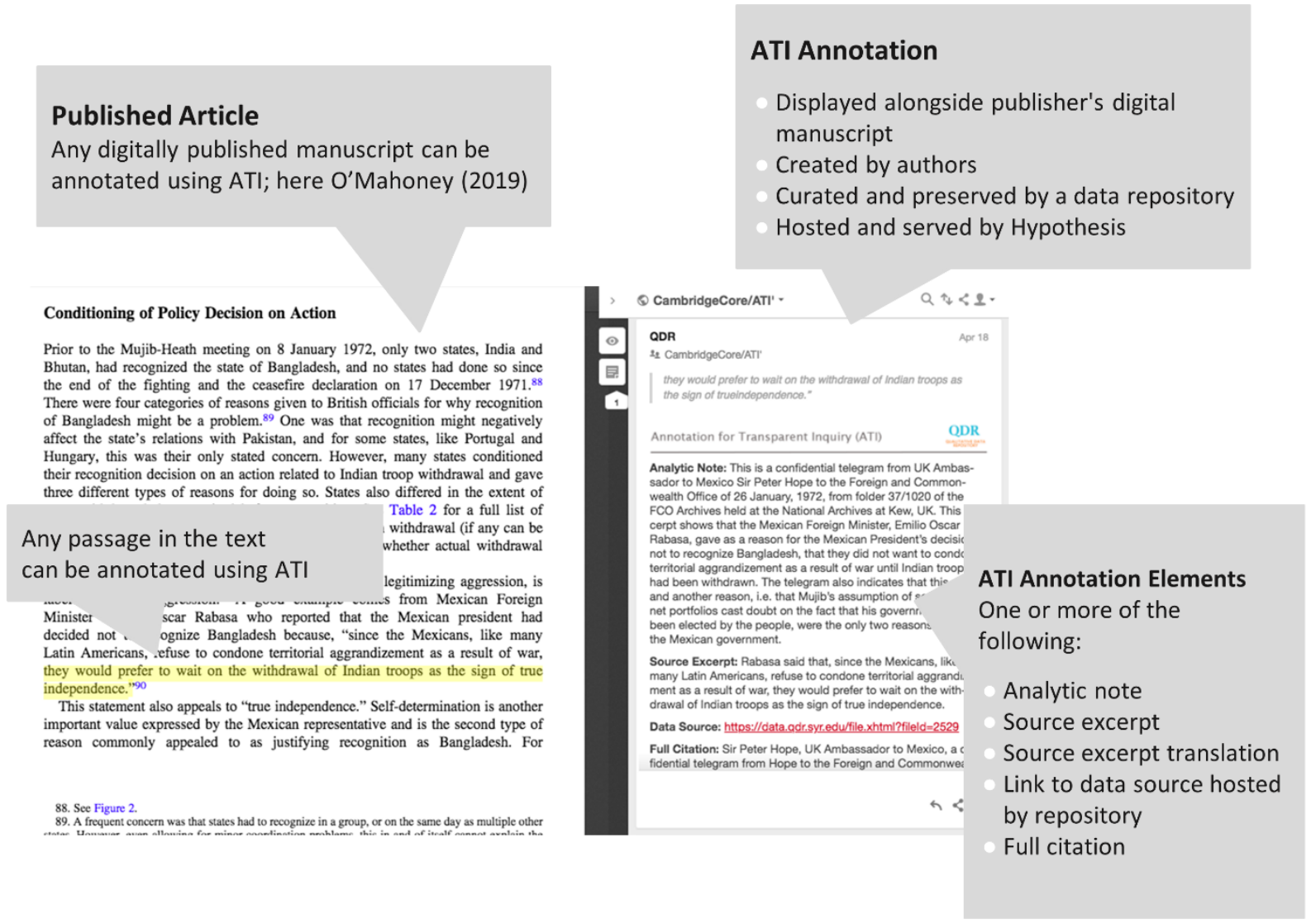

Figure 2: Figure 2: ATI at a Glance: How a passage in the text of an article annotated with ATI appears to readers

${ }^{3}$ As of this writing, QDR is the only repository that offers support for using ATI. Since all technology used is free, open source, and based on open standards, other venues can easily use it to support authors as well. 
Annotations are displayed on the same publisher web page (or PDF) as the text of the digitally published article or book that they accompany, and can be used by any publisher and on any publishing platform. Annotations (and underlying data sources) are curated and preserved by a repository where readers can further investigate them, and potentially access them for reuse (for instance, to evaluate the relevant publication, to answer new questions, or for teaching). Immediately proximate to the text and instantly available to readers, annotations enhance scholarship in the multiple ways noted above, all without increasing word-count.

As the contributions to this symposium show, ATI can be used in many ways to increase the transparency of multiple types of qualitative and multi-method research. ATI annotations can help authors to show the data that underlie their claims or to offer details about how they collected or analyzed the data (as noted above), to discuss reflexivity and positionality, or to provide additional context for a case study. ATI is thus a versatile approach to making qualitative research transparent (developing a methodological appendix is another). of course, as some symposium contributors note (and we consider next), scholarly use of ATI can have positive consequences that extend beyond transparency. Moreover, the technology used by ATI, open web annotations, has broader applications: it can be used to comment on or carry out peer review of digital manuscripts, and can facilitate teaching and learning. Nonetheless, the specific goal of using ATI is to enhance the transparency of qualitative and multi-method work.

\section{ATI's Potential}

As the contributions to this symposium highlight, ATI has several distinctive advantages as an approach to transparency, and produces various broader benefits for research. Myrick suggests that anticipating the use of ATI encouraged her and her co-author to approach the collection and interpretation of evidence in a more methodical way than they otherwise might have done. Also, as Mayka and Myrick both note, ATI helps authors to "invite readers in" to the social world of study by enabling them to provide rich description and employ participants' own words as evidence. Simultaneously, ATI enables scholars to pursue openness ethically by helping them protect their research participants (as the data underlying ATI annotations are stored in a trusted repository). 
Authors also found that ATI increases research integrity: by helping scholars to identify, and encouraging them to resolve, contradictions between the argument they are developing and their evidence - i.e., by holding them accountable to their data - ATI helps them to overcome "motivated reasoning," increasing the accuracy of their assertions and inferences (Mayka, Milonopoulos, and Siewert). Moreover, contributors suggest that ATI increases the accessibility of their scholarship: by acting as a digital exoskeleton, annotations can provide technical details of the method employed in a manuscript, increasing the work's evaluability and its utility to readers interested in learning how the relevant method works. Further, because of annotations' proximity to the text to which they relate, they are easier to access than other vehicles for transparency (e.g., footnotes and methodological appendices), and viewing them is less disruptive to the reading experience; indeed, readers who are mainly curious about a work's core findings and insights can hide the annotations completely (Milonopoulos, Myrick, Siewert). In aggregate, symposium contributions demonstrate that ATI is an adaptable, flexible approach to transparency that can be useful in many types of qualitative inquiry.

Of course, given ATI's novelty, challenges remain. Various contributors (e.g., Mayka and Siewert) highlight the time they dedicated to creating annotations and thus did not dedicate to other career-enhancing activities. There is also consensus on the importance of establishing a solid workflow to facilitate ATI's integration into research processes (Mayka, Myrick, and Siewert).4 Relatedly, some contributors (e.g., Mayka) struggled to identify the best point in the writing process at which to annotate, highlighting how early annotation can be helpful yet also distracting and inefficient, as some initial annotations may be cut through the editing and review process. Likewise, contributors grappled with what to annotate - and how to determine if they are annotating "too little" or "too much" (given the absence of consensual norms regarding the use of ATI; Milonopoulos); Siewert offers a promising set of criteria authors can use to evaluate and calibrate their use of ATI. Contributors also note how uncertainty about whether and how journals will integrate annotations into the peer review

\footnotetext{
${ }^{4}$ QDR has received funding from the National Science Foundation to develop a tool to help scholars engage in ATI and smooth their workflows.
} 
and publication process complicates composing annotations (Myrick). We agree that many key questions about ATI and its use remain unanswered. As the approach becomes more widely adopted, norms for its effective use in multiple types of qualitative and multi-method inquiry will need to emerge. We hope this symposium represents a step toward that goal.

\section{Conclusion}

Qualitative research makes significant contributions to political science. Nonetheless, scholars who conduct qualitative inquiry sometimes have difficulty convincing other researchers of the rigor and relevance of their work. In part, this challenge arises because the meticulous research procedures and practices that scholars of qualitative work employ to generate and analyze their data remain largely invisible; this complicates the assessment of the evidentiary value of the data and the quality of the analysis. At the core of these difficulties is a fundamental mismatch between the way in which scholars who conduct qualitative research build arguments and deploy evidence, on the one hand, and the way in which social science scholarship is represented in articles and books on the other.

ATI provides scholars who conduct qualitative inquiry with a way to reveal the power of their research, make it more understandable, and facilitate its careful evaluation. ATI does so by offering authors a platform on which to discuss in detail how they generated and analyzed their rich data; to demonstrate how those data support their claims; to describe the evidentiary value of their data; and to make those data available (when this can be done ethically and legally). In short, ATI opens a window on the inferential soul of qualitative inquiry.

By inviting and broadening access to key insights about qualitative methods, to the knowledge that qualitative and multi-method scholarship produces, and to qualitative data, ATI encourages intellectual democratization and addresses inequities in data access. Further, ATI offers a fresh approach to transparency and a new way of thinking about its goals. Because renewed emphasis on transparency in the last few decades was catalyzed by, and remains linked to, the inability to replicate or reproduce empirical scholarship (i.e., the "replication crisis"), a central goal of many transparency initiatives has been identifying bad actors. ATI, by contrast, lifts up good actors. Rather than facilitating the identification of intentional error 
in completed scholarship, ATI encourages ethical research practices by helping scholars to make their work more transparent as they produce it. This normative orientation sends a subtle but powerful signal and - in combination with the data protection, preservation, and promotion that ATI encourages and facilitates - makes ATI an approach to transparency around which scholars from diverse perspectives can rally. As such, ATI holds the potential to help bridge the divides over transparency that have developed among scholars who generate and analyze qualitative data, contributing to the health of contemporary social science.

Acknowledgements: We are extremely grateful to the two scholars who reviewed this piece and the other contributions to the symposium for their incisive and productive comments, which made our contribution and the symposium as a whole much stronger. We likewise thank the symposium contributors whose work inspired ours. This article is based upon work supported by the National Science Foundation under Grant No. 1823950 and Grant No. 1946272.

\section{References}

Bolsen, Toby, Thomas J. Leeper, and Matthew A. Shapiro. 2014. "Doing What Others Do: Norms, Science, and Collective Action on Global Warming." American Politics Research 42 (1): 6589. https://doi.org/10.1177/1532673X13484173

Elman, Colin, Diana Kapiszewski, and Arthur Lupia. 2018. "Transparent Social Inquiry: Implications for Political Science." Annual Review of Political Science 21 (1): 29-47. https://doi.org/10.1146/annurev-polisci-091515-025429

Karcher, Sebastian, Dessislava Kirilova, and Nicholas Weber. 2016. "Beyond the Matrix: Repository Services for Qualitative Data." IFLA Journal 42 (4): 292-302. https://doi.org/10.1177/0340035216672870

Mannheimer, Sara, Amy Pienta, Dessislava Kirilova, Colin Elman, and Amber Wutich. 2019. "Qualitative Data Sharing: Data Repositories and Academic Libraries as Key Partners in Addressing Challenges." American Behavioral Scientist 63 (5): 643-664. https://doi.org/10.1177/0002764218784991

Martineau, Andrew. 2019. "Reinforcing the 'Crumbling Infrastructure of Legal Research' 
Through Court-Authored Metadata." Law Libr. J. (forthcoming). https://papers.ssrn.com/abstract=3389843

Miguel, E., C. Camerer, K. Casey, J. Cohen, K. M. Esterling, A. Gerber, R. Glennerster, et al. 2014. "Promoting Transparency in Social Science Research." Science 343 (6166): 30-31. https://doi.org/10.1126/science.1245317

O'Mahoney, Joseph. 2019. “Data for: Making the Real: Rhetorical Adduction and the Bangladesh Liberation War." Qualitative Data Repository. https://doi.org/10.5064/F6M2H9VQ

Sawyer, Steve, and Mohammad Jarrahi. 2014. "Sociotechnical Approaches to the Study of Information Systems." In Computing Handbook, edited by Heikki Topi and Allen Tucker, 3rd ed. Boca Raton, FL: Chapman and Hall/CRC.

Snyder, Jack. 2015. 'Data for: 'Russia: The Politics and Psychology of Overcommitment,' in: The Ideology of the Offensive: Military Decision Making and the Disasters of 1914." QDR Main Collection. https://doi.org/10.5064/F6KW5CXS

Wilkinson, Mark D., Michel Dumontier, IJsbrand Jan Aalbersberg, Gabrielle Appleton, Myles Axton, Arie Baak, Niklas Blomberg, et al. 2016. "The FAIR Guiding Principles for Scientific Data Management and Stewardship." Scientific Data 3 (March): 160018. https://doi.org/10.1038/sdata.2016.18 